\title{
Estimation of single station interfrequency receiver bias using GPS-TEC
}

\author{
F. Arikan, ${ }^{1}$ H. Nayir, ${ }^{2}$ U. Sezen, ${ }^{1}$ and O. Arikan $^{3}$
}

Received 23 November 2007; revised 17 March 2008; accepted 3 June 2008; published 29 July 2008.

[1] Dual-frequency Global Positioning System (GPS) receivers present a plausible and cost-effective way of computing Total Electron Content (TEC). For accurate estimates of TEC, frequency-dependent satellite and receiver instrumental biases should be removed from GPS measurements properly. Although instrumental satellite bias values are widely available through the internet from various International GPS Service (IGS) analysis centers, receiver biases (also known as differential code biases or interfrequency biases) are provided only for a very few GPS stations and a select number of days. This makes it very difficult to compute TEC for a single station. In this study, an online, single station receiver bias estimation algorithm, IONOLAB-BIAS, is developed and implemented to obtain daily and monthly averages of receiver bias. The algorithm is successfully applied to both quiet and disturbed days of the ionosphere for stations positioned in high-latitude, midlatitude, and equatorial regions. The receiver bias estimates are compared with two of the basic methods in the literature that can be applied off-line, and also with the receiver bias values provided from the IGS centers for a select number of stations. It is observed that IONOLAB-BIAS is in excellent accordance with the sparse estimates from the IGS centers for all ionospheric states and regions.

IONOLAB-BIAS has a high potential to be an alternative receiver bias computation algorithm with its ease of implementation and accurate estimates for any single station GPS-TEC.

Citation: Arikan, F., H. Nayir, U. Sezen, and O. Arikan (2008), Estimation of single station interfrequency receiver bias using GPS-TEC, Radio Sci., 43, RS4004, doi:10.1029/2007RS003785.

\section{Introduction}

[2] Total Electron Content (TEC) is a key parameter in the investigation of spatial and temporal structure and variability of the ionosphere. TEC is defined as the line integral of electron density along a ray path or as a measure of the total number of electrons along a path of the radio wave. In recent years, Global Positioning System (GPS) dual frequency signals have been widely used to estimate both regional and global TEC values. TEC can be derived from the delay of the traveling time of the transmitted dual-frequency GPS signals, recorded

\footnotetext{
${ }^{1}$ Department of Electrical and Electronics Engineering, Hacettepe University, Beytepe, Ankara, Turkey.

${ }^{2}$ Department of Microwave and System Technologies, Aselsan Inc., Yenimahalle, Ankara, Turkey.

${ }^{3}$ Department of Electrical and Electronics Engineering, Bilkent University, Bilkent, Ankara, Turkey.
}

Copyright 2008 by the American Geophysical Union. 0048-6604/08/2007RS003785 at the earth-based receivers. Yet, variation of the ionospheric refractive index with frequency is a major source of error in computation of group delay and phase advance of GPS observables. Absolute TEC can be measured from the differential delay of the GPS code on the two GPS frequencies. For both GPS precise positioning applications and for accurate TEC estimation the effect of interfrequency satellite and receiver differential delay biases should be removed from GPS measurements [Coco et al., 1991; Warnant, 1997; Otsuka et al., 2002; Chen et al., 2004; Brunini et al., 2005]. The receiver biases are also referred to as receiver instrumental bias, receiver differential bias, receiver offset, differential code bias (DCB) and interfrequency bias (IFB).

[3] Historically, the interfrequency biases are considered to be instrumental and they are thought to be due to the delays caused by the analog hardware of satellite and receiver [Lanyi and Roth, 1988; Warnant, 1997; Goodwin and Breed, 2001]. With the assumption that the calibration differences are due to instrumentation, the interfrequency biases are modeled to be temperature- and hardware- 
dependent [Bishop et al., 1996; Warnant, 1997]. The differential code biases are investigated by various researchers. Some methods are developed to obtain TEC and differential biases by considering more than one station in their computation and model TEC on the double differences of GPS recordings [HernandezPajares et al., 2004; Makela et al., 2001; Warnant, 1997; Sardon et al., 1994]. For single-station TEC and differential receiver bias estimates, there are two basic approaches that can be found in the literature. First group of studies models TEC by a polynomial of coordinates in Earth-Sun reference system. Both satellite and receiver biases are also included in the model. The polynomial coefficients and biases being the unknowns, the observations form a linear system of equations that is solved by least squares method [Lanyi and Roth, 1988; Coco et al., 1991; Jakowski et al., 1996; Warnant, 1997; Lin, 2001; Kee and Yun, 2002; Otsuka et al., 2002; Chen et $a l ., 2004]$. In the second group of studies, for a selected measurement time, TEC is computed from different satellites over a certain angle of elevation, and the computed TEC values are considered be close to each other. This proximity is found by calculating standard deviation of TEC obtained from all satellites. To obtain the optimum receiver bias value, trial receiver biases are used in TEC computation and the receiver bias that minimizes the standard deviation is chosen as the receiver bias value for that GPS station [Ma and Maruyama, 2003; Zhang et al., 2003]. Both of the above methods can be applied to estimate differential receiver biases for a single station, yet they have to be used off-line.

[4] Differential satellite and receiver biases can also be obtained from internet for a few number of GPS stations from International GPS Service (IGS) analysis centers, namely, the Center for Orbit Determination in Europe (CODE) University of Berne, Switzerland; Jet Propulsion Laboratory (JPL) Pasadena, CA, USA; European Space Operations Center (ESOC) of European Space Agency (ESA), Darmstadt, Germany; and gAGE/UPC of Polytechnical University of Catalonia, Barcelona, Spain. Global Ionospheric TEC maps (GIM) and interfrequency bias solutions of these analysis centers and are available at the web sites ftp://igs.ensg.ign.fr/pub/igs/iono or ftp:// cddis.gsfc.nasa.gov/gps/products/ionex/ in the form of IONosphere Map EXchange Format (IONEX) files. Most of the IGS receiver differential biases provided in the IONEX files are monthly averages of daily values and do not represent the daily variations. The algorithms to compute the receiver bias values are not clearly explained in the literature, and thus the results can not be duplicated by other users. Also, the values provided for DCBs in IONEX files from various centers are not always in accordance with each other [Brunini et al., 2005].
[5] In the work of Grejner-Brzezinska et al. [2004], the receiver DCBs are computed using BERNESE software. Although a value is obtained for receiver DCB using the BERNESE software, the computation method is not disclosed to the users in the manual. The temporal and spatial variability of TEC biases are investigated in detail by Brunini et al. [2005]. It is concluded that bias estimates suffer from the same shortcomings of GPSTEC assumptions and equatorial regions need more attention in modeling and computation of TEC. From the above discussion and from Kee and Yun [2002], it can be summarized that receiver differential biases have to be included in the TEC computation model for calibration purposes and there is a certain need to develop an online bias estimator that can be applied to any single station for any ionospheric state and compute TEC along with DCBs.

[6] In this study, a new algorithm for the computation of single station receiver differential bias is introduced. The new algorithm uses the model of slant TEC (STEC) computed from difference in GPS observables. The vertical TEC (VTEC) is obtained from IGS-IONEX files and the conversion from VTEC to STEC is done by the mapping function explained in section 2. The receiver bias is extracted from the equation for de-noised difference of pseudorange and VTEC. The algorithm is originally developed by Nayir [2007b] and presented by Nayir [2007a]. The DCB bias estimates obtained from this method will be called as IONOLAB-BIAS and they are currently used in IONOLAB-TEC available at http://www.ionolab.org online. The IONOLAB-BIAS estimates are compared with the polynomial VTEC model, the minimization of standard deviation of VTEC method, and the receiver DCB estimates from the IGS centers, both for quiet and disturbed days of the ionosphere and for stations from all ionospheric regions. It is observed that IONOLAB-BIAS provides a strong alternative to online single station DCB estimation and it is very robust for various ionospheric states and regions.

[7] In section 2, the model for the GPS observables and the computation of TEC is provided. The IONOLAB-BIAS is described in section 3. The polynomial model of $V T E C$ and minimization of standard deviation of VTEC methods are reviewed briefly in section 4 . The comparison of these three methods and also the comparison with IGS DCB estimates are provided in section 5.

\section{Model for GPS Observables}

[8] The receivers at GPS stations record signals transmitted at two L-band frequencies namely, $f_{1}$ at 1575.42 $\mathrm{MHz}$, and $f_{2}$ at $1227.60 \mathrm{MHz}$. The time delay which occurs while these signals are propagating through the ionosphere are converted to 'pseudo-ranges' and 
recorded as $P_{1}$ and $P_{2}$ signals. The carrier phase delay measurements on the $f_{1}$ and $f_{2}$ coherent frequencies are also recorded as $L_{1}$ and $L_{2}$, respectively. The delayed and phase shifted signals are recorded in a special format called Receiver Independent Exchange Format (RINEX). The time delay of signals are converted to pseudo-range values and the phase shifts are recorded as phase delays in the receivers [Leick, 2004]. The standard model for pseudo-range recordings for two frequencies $f_{1}$ and $f_{2}$ are as follows:

$P_{1, u}^{m}=p_{u}^{m}+c\left(\delta t_{u}-\delta t^{m}\right)+d_{\text {trop }, u}^{m}+d_{\text {ion } 1, u}^{m}+c\left(\varepsilon_{1}^{m}+\varepsilon_{1, u}\right)$

$P_{2, u}^{m}=p_{u}^{m}+c\left(\delta t_{u}-\delta t^{m}\right)+d_{\text {trop }, u}^{m}+d_{\text {ion } 2, u}^{m}+c\left(\varepsilon_{2}^{m}+\varepsilon_{2, u}\right)$

where the subscript $u$ denotes the receiver station index; the superscript $m$ denotes the satellite index. $p$ is the actual range between satellite and receiver, $\delta t_{u}$ and $\delta t^{m}$ are the clock errors for the receiver and satellite, respectively. $d_{\text {trop }}$ and $d_{i o n}$ are the troposphere and ionosphere group delays, respectively. $\varepsilon^{m}$ and $\varepsilon_{u}$ are the frequency-dependent satellite and receiver biases [Leick, 2004]. The model for GPS recordings also include antenna, pattern and noise errors, yet since those are assumed to be the same for both frequencies, usually they are not spelled out in the model equations for TEC [Lanyi and Roth, 1988]. $c$ is the speed of light in vacuum. These measurements are recorded usually every $30 \mathrm{~s}$ and thus, if a receiver records for every instant, there are $2 \times 60 \times 24=2880$ samples for each observable.

[9] The difference of equations (1) and (2) is called the geometry free linear combination of pseudo-range because the actual range $p$ is eliminated as:

$$
\begin{aligned}
P_{4, u}^{m}= & P_{2, u}^{m}-P_{1, u}^{m}=d_{i o n 2, u}^{m}-d_{i o n 1, u}^{m}+c\left(\varepsilon_{2}^{m}-\varepsilon_{1}^{m}\right) \\
& +c\left(\varepsilon_{2, u}-\varepsilon_{1, u}\right)
\end{aligned}
$$

The tropospheric contribution $d_{\text {trop }, u}^{m}$ in equations (1) and (2) and any other source of error are also eliminated since they are not a function of frequency. Using satellite and receiver biases for $f_{1}$ and $f_{2}$ frequency signals, interfrequency or differential code biases (DCBs) are defined for the satellite and receiver as follows Leick [2004]:

$$
\begin{aligned}
& D C B^{m}=\varepsilon_{1}^{m}-\varepsilon_{2}^{m} \\
& D C B_{u}=\varepsilon_{1, u}-\varepsilon_{2, u}
\end{aligned}
$$

where $D C B^{m}$ and $D C B_{u}$ are the differential code biases for the satellite and receiver, respectively.
[10] Similar equations can be written for phase delay observations $L_{1, u}^{m}$ and $L_{2, u}^{m}$ as Leick [2004]:

$$
\begin{aligned}
L_{1, u}^{m}= & \lambda_{1} \Phi_{1, u}^{m}=p_{u}^{m}+c\left(\delta t_{u}-\delta t^{m}\right)+\lambda_{1} \Phi_{i o n 1, u}^{m} \\
& +\lambda_{1} \Phi_{\text {trop }, u}^{m}-c\left(\varepsilon_{1}^{m}+\varepsilon_{1, u}\right)+\lambda_{1} N_{1}^{m} \\
L_{2, u}^{m}= & \lambda_{2} \Phi_{2, u}^{m}=p_{u}^{m}+c\left(\delta t_{u}-\delta t^{m}\right)+\lambda_{2} \Phi_{i o n 2, u}^{m} \\
& +\lambda_{2} \Phi_{\text {trop }, u}^{m}-c\left(\varepsilon_{2}^{m}+\varepsilon_{2, u}\right)+\lambda_{2} N_{2}^{m}
\end{aligned}
$$

where $\lambda_{1}$ and $\lambda_{2}$ are the wavelengths corresponding to $f_{1}$ and $f_{2}$ frequencies, $\Phi_{1, u}^{m}$ and $\Phi_{2, u}^{m}$ are the recorded the phase delays corresponding to $f_{1}$ and $f_{2}$ frequencies, respectively. $\Phi_{i o n 1, u}^{m}$ and $\Phi_{i o n 2, u}^{m}$ are the ionospheric phase delays corresponding to $f_{1}$ and $f_{2}$ frequencies, respectively. $N_{1}^{m}$ and $N_{2}^{m}$, denote the initial phase ambiguity corresponding to $f_{1}$ and $f_{2}$ frequencies, respectively, for the $m^{\text {th }}$ satellite. Finally, $\Phi_{\text {trop }, u}^{m}$ is the phase delay due to troposphere.

[11] The difference of equations (6) and (7) is called the geometry free linear combinations of phase delay and is given as

$$
\begin{aligned}
L_{4, u}^{m}= & \lambda_{1} \Phi_{1, u}^{m}-\lambda_{2} \Phi_{2, u}^{m}=\lambda_{1} \Phi_{i o n 1, u}^{m}-\lambda_{2} \Phi_{i o n 2, u}^{m} \\
& +c\left(D C B^{m}\right)+c\left(D C B_{u}\right)+\Delta N^{m}
\end{aligned}
$$

and $\Delta N^{m}$ in equation (8) is defined as

$$
\Delta N^{m}=\lambda_{1} N_{1}^{m}-\lambda_{2} N_{2}^{m}
$$

Using the approximation given by Liao [2000] and Leick [2004]

$$
d_{i o n, u}^{m}=-\Phi_{i o n, u}^{m} \frac{c}{f} \approx A \frac{S T E C_{u}^{m}}{f^{2}}
$$

where $A=40.3 \mathrm{~m}^{3} / \mathrm{s}^{2}$ and $\operatorname{STEC}_{u}^{m}$ denotes the total electron content on the slant ray path combining the receiver $u$ and the satellite $m$. Using equation (10) in equations (3) and (8), the following expressions for the geometry free combinations are obtained [Leick, 2004; Komjathy, 1997; Nayir, 2007b]

$$
P_{4, u}^{m}=A\left(\frac{f_{1}^{2}-f_{2}^{2}}{f_{1}^{2} f_{2}^{2}}\right) S T E C_{u}^{m}-c\left(D C B^{m}+D C B_{u}\right)
$$

$$
L_{4, u}^{m}=A\left(\frac{f_{1}^{2}-f_{2}^{2}}{f_{1}^{2} f_{2}^{2}}\right) S T E C_{u}^{m}-c\left(D C B^{m}+D C B_{u}\right)+\Delta N^{m}
$$

[12] For a selected measurement time, Slant Ray Total Electron Content (STEC) can be calculated using either pseudorange or carrier phase data from each satellite. 
STEC calculated from equation (11) is noisy and open to multipath effects:

$\operatorname{STEC}_{u}^{m}(n)=\frac{1}{A}\left(\frac{f_{1}^{2} f_{2}^{2}}{f_{1}^{2}-f_{2}^{2}}\right)\left[P_{4, u}^{m}(n)+c\left(D C B^{m}+D C B_{u}\right)\right]$

where the index $n$ denotes the time sample, and $1 \leq$ $n \leq N$.

[13] In order to compute STEC from equation (12), the initial phase ambiguity $\Delta N^{m}$ needs to be resolved. In the works of Nayir [2007b] and Nayir et al. [2007], the following baseline method is used: First, a baseline, $B$, for each connected arc is obtained by differentiating pseudorange and phase measurements as

$$
B=\frac{1}{N_{m e}} \sum_{n_{m e}=1}^{N_{m e}}\left(P_{4, u}^{m}\left(n_{m e}\right)-L_{4, u}^{m}\left(n_{m e}\right)\right)
$$

where $N_{m e}$ is the number of measurements in a connected phase arc. Then, the slant TEC can be computed by inserting $B$ into the phase equation (12) and STEC can be extracted as

$$
\begin{aligned}
\operatorname{STEC}_{u}^{m}(n)= & \frac{1}{A}\left(\frac{f_{1}^{2} f_{2}^{2}}{f_{1}^{2}-f_{2}^{2}}\right)\left(B+L_{4, u}^{m}(n)\right. \\
& \left.+c\left(D C B_{u}+D C B^{m}\right)\right)
\end{aligned}
$$

In the above equations, $u$ and $m$ denote the receiver and satellite id's, respectively, $n$ is the measurement time. $L_{4}$ is the geometry free linear combination of carrier phase data and $B$ is the baseline value that is defined in equation (14). $D C B_{u}$ and $D C B^{m}$ are the receiver and satellite differential code biases respectively. In equation (15), $P_{4}$ is the pseudorange geometry free linear combination for dual frequency GPS signals. Each cycle slip or phase disconnection starts another baseline calculation. Once the slant TEC is computed, the vertical TEC, VTEC, can be obtained using thin shell approximation of Single Layer Ionosphere Model (SLIM) as

$$
\operatorname{VTEC}_{u}^{m}(n)=\operatorname{STEC}_{u}^{m}(n) / M\left(\epsilon_{m}(n)\right)
$$

where

$$
M\left(\epsilon_{m}(n)\right)=\left[1-\left(\frac{R \cos \epsilon_{m}(n)}{R+h}\right)^{2}\right]^{-1 / 2}
$$

is called the mapping function [Arikan et al., 2003, 2004]. $\varepsilon$ is the satellite elevation angle. $R$ is the earth radius of $6,378.137 \mathrm{~km}$ and $h$ is the ionospheric shell height of $428.8 \mathrm{~km}$ from Schaer [1999]. The choice of ionospheric shell height is widely discussed by Nayir [2007b] and Nayir et al. [2007]. As it can be seen from the above summary, TEC is a derived quantity and GPSTEC is modeled to include interfrequency biases of satellite and receiver hardware. The satellite ephemeris data and satellite biases are widely available in IONEX files from IGS centers. $\varepsilon_{m}(n)$ can be computed from satellite-receiver geometry using satellite ephemeris data. In the following sections, three alternative bias estimation methods are discussed and estimates are compared with each other for various ionospheric states and regions.

\section{IONOLAB-BIAS Method}

[14] The IONOLAB-BIAS is a new online estimation algorithm for receiver differential bias [Nayir, 2007b]. The general outline of the algorithm can be summarized as follows: (1) VTEC values to be used in equation (16) are obtained from GIM for every two hours. (2) $P_{4, u}^{m}$ are obtained from the RINEX files. (3) The satellite ephemeris data and DCB values are obtained from IONEX files. (4) Using the above data in equation (16), STEC is computed. (5) From equation (13), the $D C B_{u}$ is extracted for one instant of time, for one GPS station, and for one satellite as

$$
\begin{aligned}
\operatorname{DCB}_{u}(n)= & \left(\frac{A\left(f_{1}^{2}-f_{2}^{2}\right)}{c f_{1}^{2} f_{2}^{2}}\right) \underbrace{\operatorname{VTEC}(n) M\left(\epsilon_{m}(n)\right)}_{S T E C_{u}^{m}(n)} \\
& -\frac{1}{c} P_{4, u}^{m}(n)+D C B^{m}
\end{aligned}
$$

As mentioned in the previous section, $P_{4, u}^{m}(n)$ can be computed every $30 \mathrm{~s}$. Yet, GIM are updated every two hours. An interpolation of IGS-TEC from GIM is necessary to apply the algorithm for periods in-between data points. $P_{4, u}^{m}(n)$ is open to multipath effects and noisy [Arikan et al., 2003, 2004; Nayir, 2007b]. In order to reduce the multipath, only the data from the satellites over $60^{\circ}$ elevation angle are included into the bias computation. Thus, the bias of those satellites over $60^{\circ}$ elevation angle are added to $P_{4, u}^{m}(n)$ in equation (13). In order to reduce the noise, a de-noising Chebyschev filter is applied to $P_{4, u}^{m}(n)+c D C B^{m}$. An example of the noisy and de-noised $P_{4, u}^{m}(n)+c D C B^{m}$ is provided in Figure 1 for Zelenchukskaya on 10 October 2003 for PRN 15.

[15] The differential bias $D C B_{u}(n)$ can be calculated for each time index $n$ using equation (18) by inserting in the interpolated IGS-TEC from GIM, filtered $P_{4, u}^{m}(n)+c$ $D C B^{m}, A$ and the frequencies $f_{1}$ and $f_{2}$. A summary of IONOLAB-BIAS computation is provided in Figure 2 for the case when the VTEC values are obtained from CODE-GIM.

[16] The $D C B_{u}$ can be computed for any duration of time with IONOLAB-BIAS and the variation with re- 

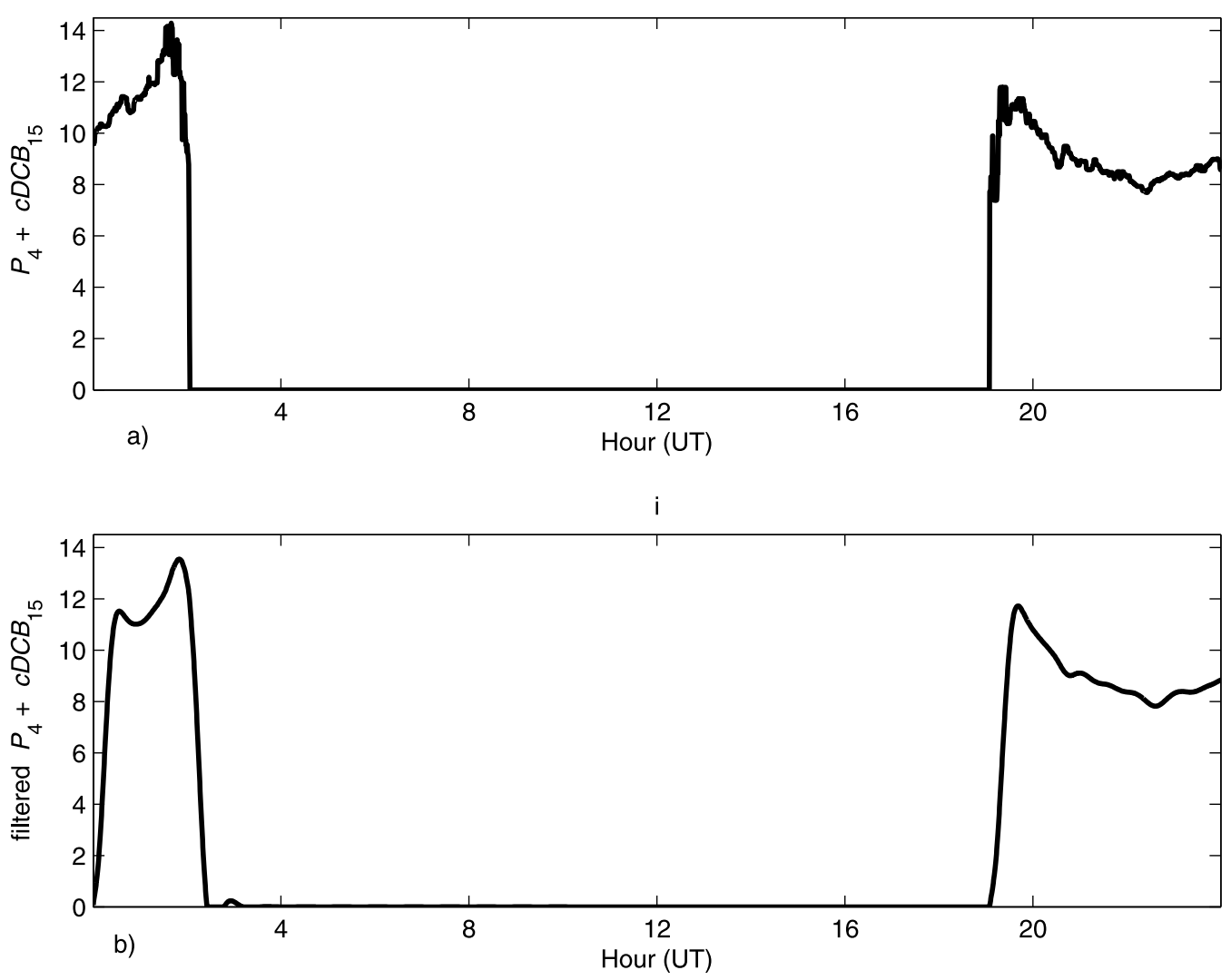

Figure 1. Denoising of $P_{4}+c D C B$ for Zelenchukskaya on 10 October 2003 for PRN 15 (a) $P_{4}+$ $c D C B$ and (b) filtered $P_{4}+c D C B$.

spect to position of satellites and day and night variability can be observed. Yet, in most IONEX files hourly and daily values are very rare and for comparison purposes, we chose to average the $D C B_{u}(n)$ values over 24-hours for daily averages. The monthly averages are obtained by taking the mean of daily DCB's over a month. The IONOLAB-BIAS is currently used in IONOLAB-TEC at http://www.ionolab.org using the Reg-Est TEC computation method given by Arikan et al. [2003, 2004, 2007] and Nayir et al. [2007]. In the following section, two alternative off-line DCB estimation methods will be briefly reviewed.

\section{Alternative DCB Estimation Methods}

[17] As it is discussed in the Introduction, there are various DCB estimation algorithms that can be found in the literature, and the most commonly used methods can be roughly grouped into two categories. In the first group, VTEC is expanded into a polynomial of coordinates and the unknown coefficients are solved using least squares along with the unknown bias values. Being one of the earliest studies in the area, we have chosen to implement the algorithm described by Lanyi and Roth [1988] for this group. The second alternative group of studies use the method of minimization of standard deviation of VTEC, and we implemented the method discussed [Ma and Maruyama, 2003] for a single station.

\subsection{Polynomial Model of VTEC Method}

[18] In this method, VTEC is modeled as a polynomial that is a function of ionospheric pierce point coordinates in a coordinate system referenced to Earth-Sun axis [Lanyi and Roth, 1988]. Ionospheric pierce point coordinates are provided by Lanyi and Roth [1988, Appendix A], using the angular definitions between the satellite and GPS receiver coordinates and ionospheric thin shell height. The slant TEC is modeled as a polynomial of angular coordinate differences as follows:

$$
\begin{aligned}
\operatorname{STEC}_{u}^{m}(n)= & o_{u}^{m}+M\left(\epsilon_{m}(n)\right)\left(c_{1}+c_{2} \bar{\phi}_{p}+c_{3} \bar{\theta}_{p}\right. \\
& \left.+c_{4} \bar{\phi}_{p}^{2}+c_{5} \bar{\phi}_{p} \bar{\theta}_{p}+c_{6} \bar{\theta}_{p}^{2}\right)
\end{aligned}
$$




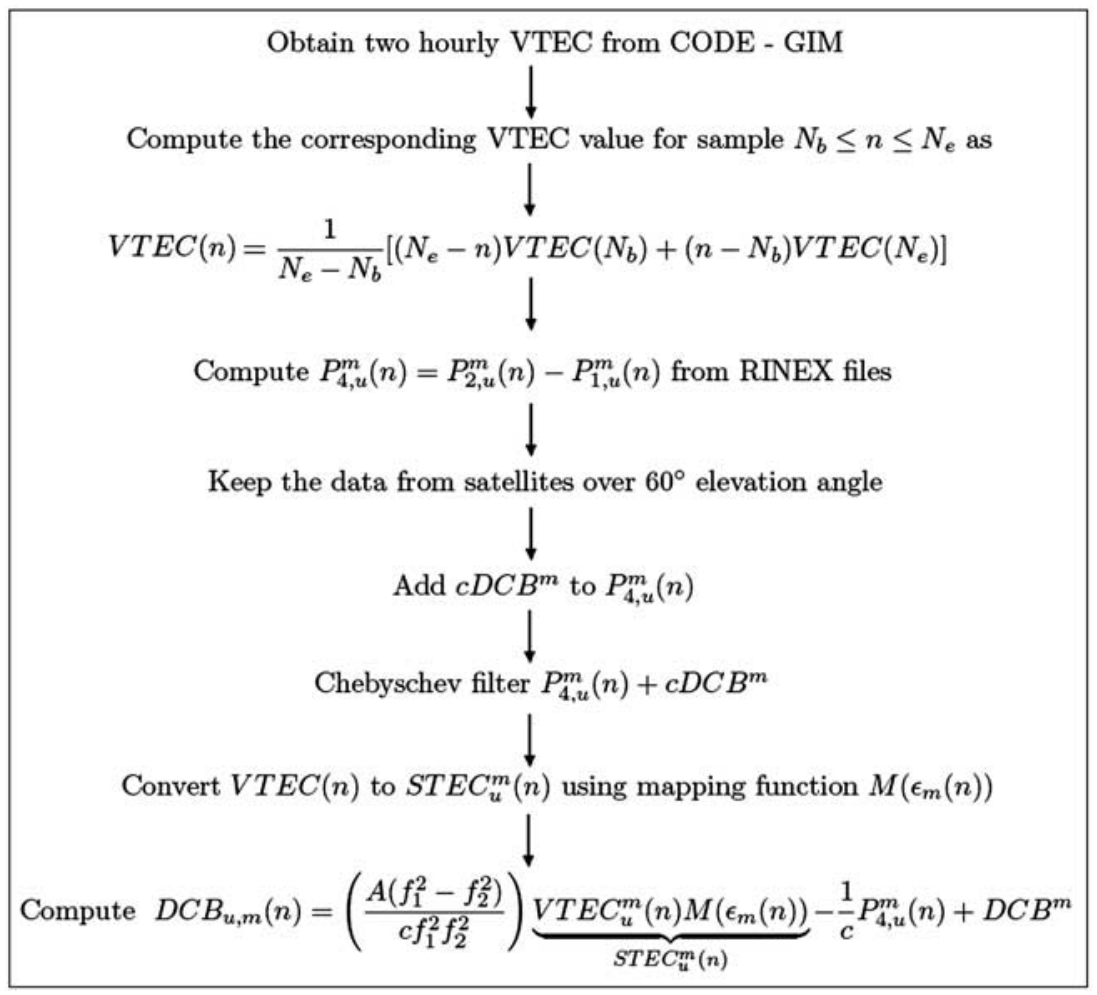

Figure 2. Flowchart of IONOLAB-BIAS method.

where $o_{u}^{m}$ denotes the sum of satellite and receiver biases where subscript $u$ and superscript $m$ denote receiver and satellite, respectively. $c_{1}$ to $c_{6}$ are the coefficients that forms VTEC polynomial. According to Lanyi and Roth [1988], these coefficients are expected to stay constant with respect to time. Also, since offset value $\left(o_{u}^{m}\right)$ occurs due to satellite and receiver hardware, it can be assumed constant for long periods. The polynomial coefficients and offset values can be obtained using a least squares approximation separately for nighttime and daytime measurement sessions [Lanyi and Roth, 1988]. The mapping function $M\left(\varepsilon_{m}\right)$ is the same as the one given in equation (2.16). It is observed that there is a difference in the bias values for nighttime and daytime measurement sessions. In later implementations of this method by Coco et al. [1991], Jakowski et al. [1996], Warnant [1997], Lin [2001], Kee and Yun [2002], Otsuka et al. [2002], and Chen et al. [2004], the polynomial model, type and degree, duration of measurement sessions and the exact number of unknowns vary. The estimates for DCB also differ as the duration of application and polynomial model changes. Therefore, we implemented the technique in a way to stay as loyal as possible to the original method of Lanyi and Roth [1988].
[19] In our implementation of Lanyi and Roth [1988], the ionospheric pierce points and shell coordinates referenced to Earth-Sun axis are computed as in Nayir [2007b]. The suggested time duration of two hours is kept as a guideline, and overlapping two hour sessions are considered in Nayir [2007b]. The first two hour period starts from 0000 and extend to 0200 . The second two hour period is chosen as overlapping with one hour with the first period and starts from 0100 and 0300 . This way all of the 24-hour data set is used. In Lin [2001], eight 3-hour sessions are used for the solution of the polynomial coefficients. Only the satellites that can be observed totally within the overlapping two hour periods are taken in to consideration. This restriction corresponded to four active satellites over the $30^{\circ}$ elevation angle range.

[20] For each satellite and time index for the chosen two hour duration, equation (19) is formed. For example, for satellite $m_{1}$ and time index $n_{1}$, the equation (19) takes the form of:

$$
\operatorname{STEC}_{u}^{m_{1}}\left(n_{1}\right)=o_{u}^{m_{1}}+M\left(\epsilon_{m_{1}}\left(n_{1}\right)\right)\left(\operatorname{VTEC}_{u}^{m_{1}}\left(n_{1}\right)\right)
$$


where

$$
\begin{aligned}
\operatorname{VTEC}_{u}^{m_{1}}\left(n_{1}\right)= & c_{1}+c_{2} \bar{\phi}_{p}^{m_{1}}\left(n_{1}\right)+c_{3} \bar{\theta}_{p}^{m_{1}}\left(n_{1}\right) \\
& +c_{4}\left[\bar{\phi}_{p}^{m_{1}}\left(n_{1}\right)\right]^{2}+c_{5} \bar{\phi}_{p}^{m_{1}}\left(n_{1}\right) \bar{\theta}_{p}^{m_{1}}\left(n_{1}\right) \\
& +c_{6}\left[\bar{\theta}_{p}^{m_{1}}\left(n_{1}\right)\right]^{2}
\end{aligned}
$$

When equations (20) and (21) are written for $M_{t}$ satellites and $N_{t}$ measurement samples, $M_{t} \times N_{t}$ equations are obtained for one observation session. Then, the total bias, $o_{u}^{m}$, and coefficients $c_{1}, c_{2}, c_{3}, c_{4}, c_{5}, c_{6}$ are solved using least squares. For every overlapping two hour period starting from 0000 and ending at 2400, a new matrix is formed indicating the solution for the $o_{u}^{m}$ for each period for each satellite. A median value is taken for each satellite over the periods that the satellite is active. Since the $o_{u}^{m}$ value is different for each satellite, satellite DCB obtained from IONEX files are removed from these median values to compute receiver DCB. Then, the median of these receiver bias values over the 24 hour period are taken to obtain a single daily receiver bias value. Once the daily differential bias values are calculated for the selected receiver, the monthly bias values are computed by averaging the daily biases over a month.

\subsection{Minimization of Standard Deviation of VTEC Method}

[21] Another alternative for receiver bias estimation is the minimization of the standard deviation of VTEC that is computed from different satellites as discussed by $M a$ and Maruyama [2003]. This method may be implemented for the measurements of a single receiver or a group of receivers. The minimization of standard deviation method assumes that the VTEC computed from each satellite in view should be equal since the measurement time and vertical path of satellite zenith are same. This assumption is valid if the satellite and receiver biases are correctly removed from GPS measurements. Also, most VTEC computation techniques assume both the spatial homogeneity of ionosphere for a wide range of elevation and azimuth angles and a temporal stationarity period of at least 5 to 15 minutes [Komjathy and Langley, 1996; Arikan et al., 2003]. In this method, a range of receiver bias values are applied and $V T E C$ is calculated for each bias selection. If the correct receiver bias is selected, the standard deviation of VTEC data from each satellite with respect to mean should be minimum [Ma and Maruyama, 2003].

[22] Our implementation of this method is very similar to the steps described by Ma and Maruyama [2003]. STEC is obtained from equation (15) using pseudorange leveled carrier phase data. Although it is reported that there exists no difference in the choice of elevation angle limits in bias estimation in Komjathy and Langley [1996], Ma and Maruyama [2003] used a weighting function in order to reduce the multipath effects. In our implementation, we did not use the sine square weighting function in VTEC computation. In order to ensure azimuthal homogeneity and reduce multipath effects, only the satellites over the elevation angle limit of $40^{\circ}$ are considered.

[23] The standard deviation of VTEC data for a measurement period is given as

$$
\sigma_{t, u}=\sum_{n=1}^{N_{t}} \sigma_{u}(n)
$$

where

$$
\sigma_{u}(n)=\sqrt{\frac{1}{M_{t}} \sum_{m=1}^{M_{t}}\left(\operatorname{VTEC}_{u}^{m}(n)-\overline{\operatorname{VTEC}}_{u}(n)\right)^{2}}
$$

and $M_{t}$ denotes the total number of satellites and $N_{t}$ is duration of the desired measurement time interval in samples. In equation (22), total standard deviation is obtained by summing the standard deviation values of each measurement sample where $N_{t}$ is selected as 24 hours for this study corresponding to 2880 GPS measurements. $\overline{V T E C}_{u}(n)$ denotes the average of all VTEC from $M_{t}$ satellites.

[24] The minimization of standard deviation of VTEC method is applied by using trial receiver bias values starting from $-30 \mathrm{~ns}$ to $30 \mathrm{~ns}$ in $0.001 \mathrm{~ns}$ steps. For each receiver bias value $V T E C$ and total standard deviation $\sigma_{t, u}$ are calculated using above formulas. The receiver bias value that results minimum total standard deviation is the correct receiver bias value. An example of the variation of total standard deviation with respect to receiver bias is provided in Figure 3 for Graz, on 7 October 2004. In Figure 3, optimum daily differential receiver bias value is chosen as $-2,833 \mathrm{~ns}$ corresponding to the minimum of the total standard deviation.

[25] The polynomial model of VTEC method and the minimization of the standard deviation of VTEC method are both off-line methods that require measurement data for at least a period of hours or days. In the following section, the three methods are applied to various stations in all regions of the ionosphere and for both quiet and disturbed days. The computed receiver bias values are compared with those available from IGS centers.

\section{Results}

[26] IONOLAB-BIAS is computed for a large number of stations in high latitude, mid-latitude and equatorial regions and for both quiet and disturbed days of the 


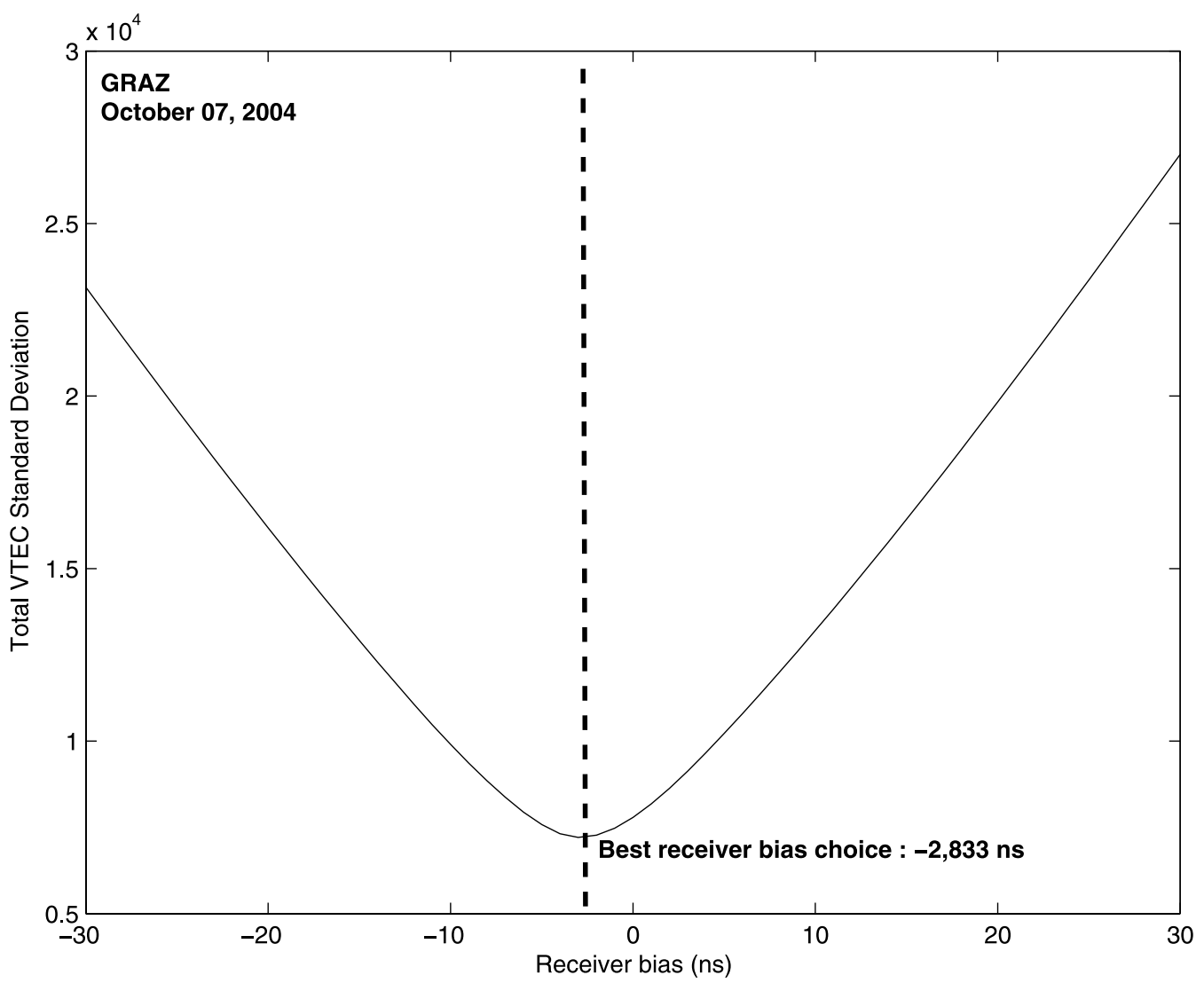

Figure 3. Differential receiver bias estimation using minimization of standard deviation of VTEC method for Graz receiver station on 7 October 2004.

ionosphere. In this section, we will report the results only for a limited subset of investigated stations and days where the IONOLAB-BIAS estimates can be compared with the alternative bias estimation methods given in section 4 and also with the IGS centers' estimates for daily and monthly averages of receiver DCB. The partial list of stations is provided in Table 1.

[27] The ionospheric quiet and disturbed days of the ionosphere are chosen according to the classification provided by the Ionospheric Dispatch Center in Europe

Table 1. List of GPS Receiver Stations

\begin{tabular}{|c|c|c|c|c|}
\hline Receiver Station & Station ID & Latitude & Longitude & Region \\
\hline Ankara, Turkey & ankr & $39.53^{\circ} \mathrm{N}$ & $32.45^{\circ} \mathrm{E}$ & Midlatitude \\
\hline Brussels, Belgium & brus & $50.47^{\circ} \mathrm{N}$ & $4.21^{\circ} \mathrm{E}$ & Midlatitude \\
\hline Delft, Netherlands & dlft & $51.59^{\circ} \mathrm{N}$ & $4.23^{\circ} \mathrm{E}$ & Midlatitude \\
\hline Graz, Austria & graz & $47.04^{\circ} \mathrm{N}$ & $15.29^{\circ} \mathrm{E}$ & Midlatitude \\
\hline Sofia, Bulgaria & sofi & $42.33^{\circ} \mathrm{N}$ & $23.23^{\circ} \mathrm{E}$ & Midlatitude \\
\hline Yerevan, Armenia & nssp & $40.13^{\circ} \mathrm{N}$ & $04.43^{\circ} \mathrm{E}$ & Midlatitude \\
\hline Zelenchukskaya, Russia & zeck & $43.17^{\circ} \mathrm{N}$ & $41.33^{\circ} \mathrm{E}$ & Midlatitude \\
\hline Arti, Russia & artu & $56.25^{\circ} \mathrm{N}$ & $58.33^{\circ} \mathrm{E}$ & High-Latitude \\
\hline Petropavlovsk, Russia & petp & $53.04^{\circ} \mathrm{N}$ & $158.36^{\circ} \mathrm{E}$ & High-Latitude \\
\hline Metsahovi, Finland & mets & $60.13^{\circ} \mathrm{N}$ & $24.41^{\circ} \mathrm{E}$ & High-Latitude \\
\hline Lae, Papua New Guinea & lae1 & $6.40^{\circ} \mathrm{S}$ & $146.59^{\circ} \mathrm{E}$ & equatorial \\
\hline Nanyang, Singapore & ntus & $1.20^{\circ} \mathrm{N}$ & $103.40^{\circ} \mathrm{E}$ & equatorial \\
\hline Cocos (Keeling) Island, Australia & coco & $12.11^{\circ} \mathrm{S}$ & $96.50^{\circ} \mathrm{E}$ & equatorial \\
\hline
\end{tabular}


Table 2. Comparison of IONOLAB-BIAS, Polynomial Model of VTEC Method, and Minimization of Standard Deviation of VTEC Method Receiver DCB Estimates for 8 October 2004

\begin{tabular}{lcccccc}
\hline Station ID & $D C B_{S}(\mathrm{~ns})$ & $D C B_{P}(\mathrm{~ns})$ & $D C B_{I}(\mathrm{~ns})$ & $D C B_{S-P}(\mathrm{~ns})$ & $D C B_{S-I}(\mathrm{~ns})$ & $D C B_{P-I}(\mathrm{~ns})$ \\
\hline zeck & -4.608 & -3.974 & -3.631 & -0.635 & -0.977 & -0.343 \\
graz & -2.873 & -1.655 & -2.422 & -1.218 & -0.452 & 0.766 \\
brus & -4.677 & -3.202 & -4.455 & -1.475 & -0.222 & 1.253 \\
nssp & -4.711 & -3.382 & -2.845 & -1.329 & -1.866 & -0.538 \\
sofi & -1.182 & -0.699 & -0.240 & -0.483 & -0.942 & -0.459 \\
mets & -10.923 & -10.273 & -11.174 & -0.650 & 0.251 & 0.901 \\
artu & -8.649 & -6.980 & -8.408 & -1.669 & -0.241 & -1.428 \\
lae1 & -12.945 & -12.954 & -7.700 & 0.009 & -5.245 & -5.254 \\
ntus & -2.638 & -6.452 & 0.344 & 3.814 & -2.982 & -6.796 \\
\hline
\end{tabular}

(IDCE) (http://www.cbk.waw.pl/rwc/idce.html). According to IDCE, 10-12 October 2003 and 6-12 October 2004 are quiet days; $27-29$ October 2003 are positively disturbed days; and 30-31 October 2003 are negatively disturbed days. Between 27 and 31 October 2003, there was a severe geomagnetic storm causing major disturbance in the ionosphere. $K p$ index rose as high as 9 and Dst index fell to $-400 \mathrm{nT}$ as given in Arikan et al. [2007]. A partial list of the studies for October 2003 storm includes Foster and Rideout [2005], Lin et al. [2005], Mitchell et al. [2005], and Yizengaw et al. [2005].

[28] An example of the daily receiver bias estimates for a quiet day 8 October 2004 for the three methods is provided in Table 2. In Table $2, D C B_{S}$ denotes the estimates from the minimization of the standard deviation of $V T E C$ method, $D C B_{P}$ stands for results of polynomial model of $V T E C$ method and $D C B_{I}$ denotes the results of IONOLAB-BIAS method. In equation (24) through (26) differences between TEC estimates are defined as

$$
\begin{aligned}
& D C B_{S-P}=D C B_{S}-D C B_{P} \\
& D C B_{S-I}=D C B_{S}-D C B_{I} \\
& D C B_{P-I}=D C B_{P}-D C B_{I}
\end{aligned}
$$

where $D C B_{S-P}$ is the receiver bias difference between the polynomial model of $V T E C$ method and minimization of standard deviation of VTEC method, $D C B_{S-I}$ is the receiver bias difference between minimization of standard deviation of VTEC method and IONOLABBIAS, $D C B_{P-I}$ is the receiver bias difference between polynomial model VTEC method and IONOLAB-BIAS. It is observed from Table 2 and from other computed differences in bias estimates for both quiet and disturbed days of the ionosphere, $D C B_{S-P}$ is small and and under $2 \mathrm{~ns}$ for most stations. The highest values of $D C B_{S-P}$ are under $4 \mathrm{~ns}$ for all the stations and days we have observed.
The investigation of $D C B_{S-I}$ and $D C B_{P-I}$ indicate that the bias estimates are similar for high latitude and midlatitude stations, yet the differences increase to $7 \mathrm{~ns}$ for equatorial stations.

[29] The estimates of IONOLAB-BIAS are also compared with those from the IGS centers, such as CODE and JPL for a number of quiet and disturbed days. An example of differences in the DCB estimates is presented in Tables 3 and 4, for CODE and JPL, respectively, for 8 October 2004. The estimates from the CODE are denoted as $D C B_{C}$, and they are obtained from CODE's monthly GNSS P1-P2 DCB Solution page (ftp.unibe.ch/ aiub/CODE/2003). In Table 3, the differences from the CODE estimates are denoted as

$$
\begin{aligned}
& D C B_{S-C}=D C B_{S}-D C B_{C} \\
& D C B_{P-C}=D C B_{P}-D C B_{C} \\
& D C B_{I-C}=D C B_{I}-D C B_{C}
\end{aligned}
$$

where $D C B_{S-C}, D C B_{P-C}$ and $D C B_{I-C}$ are the receiver bias differences between the polynomial model of $V T E C$

Table 3. Comparison of IONOLAB-BIAS, Polynomial Model of VTEC Method, and Minimization of Standard Deviation of VTEC Method Receiver DCB Estimates With Those of CODE for 8 October 2004

\begin{tabular}{ccccc}
\hline Station ID & $D C B_{C}(\mathrm{~ns})$ & $D C B_{S-C}(\mathrm{~ns})$ & $D C B_{P-C}(\mathrm{~ns})$ & $D_{I-C}(\mathrm{~ns})$ \\
\hline zeck & -3.741 & -0.867 & -0.233 & 0.110 \\
graz & -2.628 & -0.245 & 0.973 & 0.207 \\
brus & -4.536 & -0.141 & 1.334 & 0.081 \\
nssp & -2.782 & -1.929 & -0.600 & -0.063 \\
mets & -11.186 & 0.263 & 0.913 & 0.012 \\
artu & -8.422 & -0.227 & 1.442 & 0.014 \\
lae1 & -7.919 & -5.026 & -5.035 & 0.219 \\
ntus & 0.896 & -3.534 & -7.348 & -0.552 \\
\hline
\end{tabular}


Table 4. Comparison of IONOLAB-BIAS, Polynomial Model of VTEC Method, and Minimization of Standard Deviation of VTEC Method Receiver DCB Estimates With Those of JPL for 8 October 2004

\begin{tabular}{ccccc}
\hline Station ID & $D C B_{J}(\mathrm{~ns})$ & $D C B_{S-J}(\mathrm{~ns})$ & $D C B_{P-J}(\mathrm{~ns})$ & $D_{I-J}(\mathrm{~ns})$ \\
\hline zeck & -3.555 & -1.053 & -0.419 & -0.076 \\
brus & -4.432 & -0.245 & 1.230 & -0.023 \\
nssp & $-2,502$ & -2.209 & -0.880 & -0.343 \\
sofi & $-0,327$ & -0.855 & -0.372 & 0.087 \\
artu & -8.537 & -0.112 & 1.557 & 0.129 \\
lae1 & -8.818 & -4.127 & -4.136 & 1.118 \\
ntus & 1.007 & -3.645 & -7.459 & -0.663 \\
\hline
\end{tabular}

method, minimization of standard deviation of VTECmethod, and IONOLAB-BIAS and the CODE estimates, respectively. As can be observed from Table 3, the IONOLAB-BIAS estimates are in excellent accordance with those of CODE and very successful in duplicating the CODE bias for all stations.

[30] In Table 4, the estimates from JPL are denoted as $D C B_{J}$ and they are obtained from monthly averages of DCB's given in JPL IONEX files (ftp://cddisa.gsfc. nasa.gov/gps/products/ionex). In equations (30) through (32) receiver bias differences between proposed methods and results of JPL analysis center are given as

$$
\begin{aligned}
& D C B_{S-J}=D C B_{S}-D C B_{J} \\
& D C B_{P-J}=D C B_{P}-D C B_{J} \\
& D C B_{I-J}=D C B_{I}-D C B_{J}
\end{aligned}
$$

where $D C B_{S-J}, D C B_{P-J}$ and $D C B_{I-J}$ are the receiver bias differences between the polynomial model of VTEC method, minimization of standard deviation of VTEC method, and IONOLAB-BIAS estimates and the JPL monthly averages for receiver DCB, respectively. In Table 4, $D C B_{I-J}$ is significantly small for all stations and an excellent accordance is observed. IONOLAB-BIAS is very successful in duplicating the JPL bias for all stations.

[31] Another comparison of IONOLAB-BIAS estimates with those from IGS centers for both quiet and disturbed days of ionosphere and a wide variety of receiver stations is provided in Table 5 . In Table $5, D C B_{g}$ denotes the receiver DCB from IGS/gAGE and $D C B_{U}$ is the DCB estimate of UPC. Daily bias values are not listed in ESA-IONEX files for any day or any station that we have investigated. As it can be observed from Table 5, daily receiver bias estimates vary in value and consistency for IGS centers over days and stations. IONOLABBIAS can be estimated for any station and for any ionospheric state even if there is no daily bias value can be obtained from IGS centers. When IONOLABBIAS estimates are compared with those from IGS centers, the largest difference is $2.35 \mathrm{~ns}$ for coco on 11 October 2003 with JPL. For the rest of the stations and both for quiet and disturbed days, the difference in DCB's between IONOLAB-BIAS and IGS centers is under $1.5 \mathrm{~ns}$. The TEC estimates using the computed DCBs are also obtained and compared with each other. The TEC estimates of IGS centers can be obtained from their corresponding GIM and an example is provided in Figure 4 for ankr, 30 October 2003 (Figure 4a), nssp, 30 October 2003 (Figure 4b), nssp, 31 October 2003 (Figure 4c), dlft, 10 October 2003 (Figure 4d). IONOLABBIAS is estimated as discussed in section 3 and inserted into the computation of Reg-Est in the form of IONOLABTEC. Figure 4a demonstrates a case where bias estimates from IGS centers are very similar to each other and to IONOLAB-BIAS. Only UPC did not provide a receiver DCB value. It is observed from Figure $4 \mathrm{a}$, the TEC

\begin{tabular}{|c|c|c|c|c|c|c|}
\hline Station ID & Day & $D C B_{I}(\mathrm{~ns})$ & $D C B_{g}(\mathrm{~ns})$ & $D C B_{J}(\mathrm{~ns})$ & $D C B_{C}(\mathrm{~ns})$ & $D C B_{U}(\mathrm{~ns})$ \\
\hline brus & 10 Oct 2003 & -5.2514 & -5.183 & - & -5.183 & - \\
\hline dlft & 10 Oct 2003 & 26.6924 & - & - & - & - \\
\hline coco & 11 Oct 2003 & 5.2192 & 6.398 & 7.575 & 5.273 & 5.971 \\
\hline petp & 29 Oct 2003 & -5.8199 & -6.583 & -6.868 & -6.082 & -6.373 \\
\hline artu & 30 Oct 2003 & -9.9855 & -9.527 & -9.451 & -9.475 & -9.371 \\
\hline ankr & 30 Oct 2003 & 3.8798 & 3.674 & 3.601 & 3.820 & \\
\hline ntus & 30 Oct 2003 & 0.3499 & 0.897 & -0.048 & 1.715 & 1.272 \\
\hline nssp & 30 Oct 2003 & -4.3492 & - & - & - & - \\
\hline nssp & 31 Oct 2003 & -4.0979 & - & - & - & - \\
\hline brus & 31 Oct 2003 & -5.4398 & -5.212 & - & -5.252 & -5.107 \\
\hline zeck & 31 Oct 2003 & -5.1006 & -5.468 & - & -5.400 & -5.487 \\
\hline mets & 31 Oct 2003 & -11.6277 & -11.612 & - & -11.612 & - \\
\hline
\end{tabular}
estimates from JPL, CODE, UPC, IGS and IONOLAB-

Table 5. Comparison of IONOLAB-BIAS With Those From IGS-gAGE, JPL, CODE, and UPC 

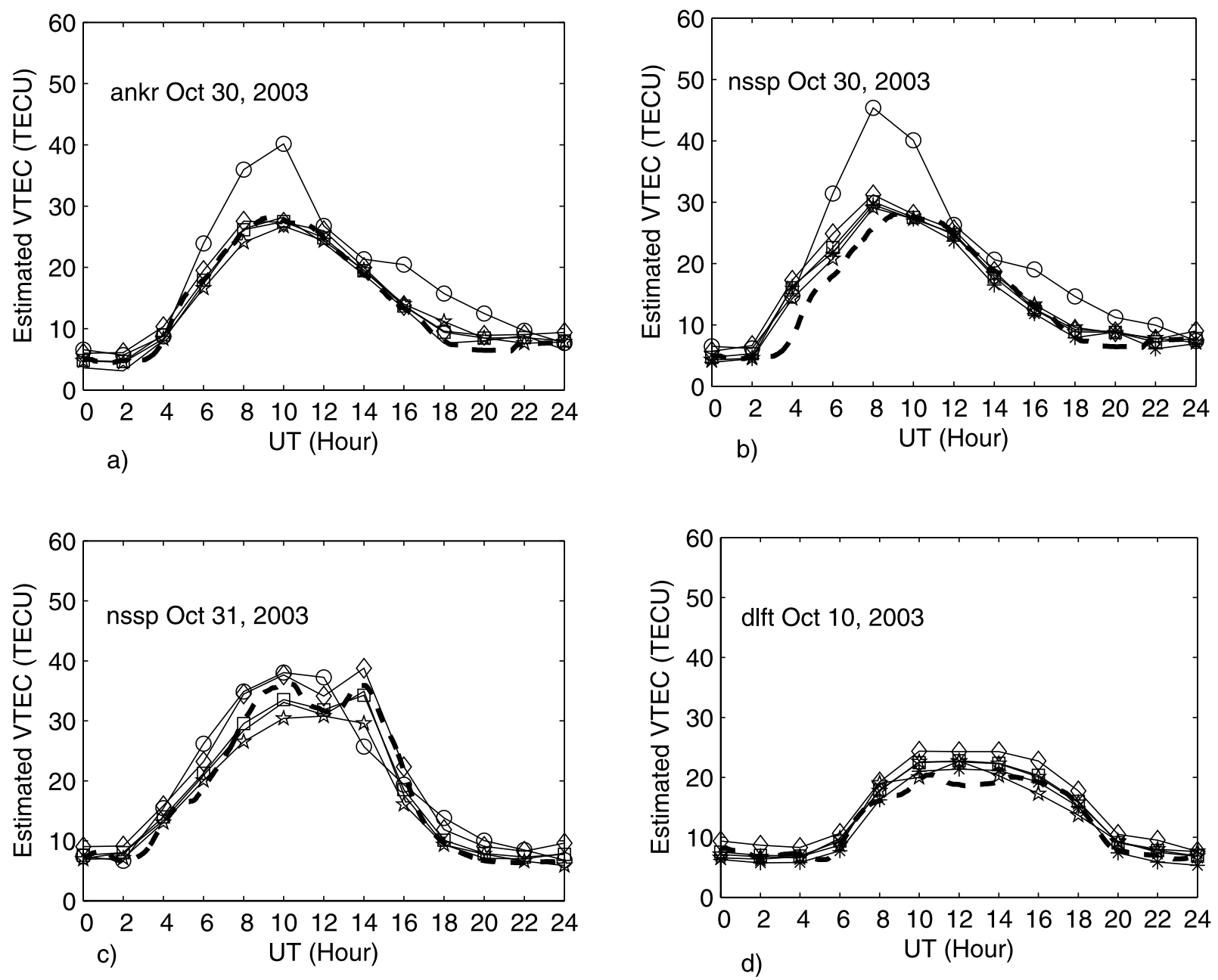

Figure 4. VTEC estimates for IONOLAB-TEC using IONOLAB-BIAS (dashed line), JPL (diamond), CODE (asterisk), ESA/ESOC (circle), UPC (star), IGS (square) (a) ankr, 30 October 2003; (b) nssp, 30 October 2003; (c) nssp, 31 October 2003; (d) dlft, 10 October 2003.

TEC are very close to each other in value. Only the TEC estimate of ESA differs from the others. In Figures $4 \mathrm{~b}-$ $4 \mathrm{~d}$, we present cases where none of the IGS centers provides daily bias values. Yet, using GIM, IONOLABBIAS can be estimated and IONOLAB-TEC using RegEst algorithm has excellent accordance with IGS centers' TEC estimates except those from ESA for 30 October 2003 for nssp.

[32] The monthly averages of the IONOLAB-BIAS estimates are compared with those from CODE and JPL and differences in estimates $D C B_{I-C, m}, D C B_{I-J, m}$ are provided in Table 6 for the month of October 2003 for stations from high-latitude, midlatitude and equatorial regions. The subscript $m$ denote that it is the monthly average value of the DCB. For the monthly averages of the receiver bias estimates, again an excellent accordance
Table 6. Comparison of Monthly Averages of IONOLABBIAS Estimates With Those From CODE and JPL for October 2003

\begin{tabular}{ccc}
\hline Station ID & $D C B_{I-C, m}(\mathrm{~ns})$ & $D C B_{I-J, m}(\mathrm{~ns})$ \\
\hline zeck & 0.076 & 0.228 \\
graz & 0.143 & - \\
brus & 0.030 & - \\
nssp & -0.031 & 0.223 \\
mets & 0.083 & - \\
artu & 0.096 & -0.319 \\
lae1 & -0.162 & 1.792 \\
ntus & -0.672 & -0.194 \\
\hline
\end{tabular}


is observed with those from both CODE and JPL. Except for the lael station in the equatorial region, the monthly averages of the differences are below $1 \mathrm{~ns}$ for all receiver stations for both CODE and JPL. This may be due to the fact that, for lae1 receiver station, JPL receiver bias estimates are obtained by using only four days in October 2003. The results presented in this section demonstrate that IONOLAB-BIAS provides a robust alternative to single station receiver differential bias estimation.

\section{Conclusion}

[33] Satellite and receiver instrumental biases are important parameters for GPS precise positioning and ionospheric TEC calculation. The differential satellite and receiver biases for a limited number of stations are available via internet through IGS analysis centers. In order to compute the receiver DCB, various methods are developed and provided in the open literature. In this study, a new algorithm, namely, IONOLAB-BIAS, is developed for single station receiver differential bias estimation. IONOLAB-BIAS is compared with two alternative offline methods in the literature and also with the DCB estimates of IGS centers for all regions and states of the ionosphere. It is observed that IONOLABBIAS is in excellent accordance with daily and monthly estimates of IGS centers where available and presents a strong alternative for single station receiver DCB estimation. IONOLAB-BIAS can be used online and provides robust estimates for DCB for stations in any ionospheric region and for both quiet and disturbed days of the ionosphere. IONOLAB-BIAS is currently in use in the computation of IONOLAB-TEC available online from http://www.ionolab.org.

[34] Acknowledgments. This project is supported by TUBITAK EEEAG grant 105E171.

\section{References}

Arikan, F., C. B. Erol, and O. Arikan (2003), Regularized estimation of vertical total electron content from Global Positioning System data, J. Geophys. Res., 108(A12), 1469, doi:10.1029/2002JA009605.

Arikan, F., C. B. Erol, and O. Arikan (2004), Regularized estimation of vertical total electron content from GPS data for a desired time period, Radio Sci., 39, RS6012, doi:10.1029/ 2004RS003061.

Arikan, F., O. Arikan, and C. B. Erol (2007), Regularized estimation of TEC from GPS data for certain mid-latitude stations and comparison with the IRI model, $A d v$. Space Res., 39, 867-874, doi:10.1016/j.asr.2007.01.082.

Bishop, G., A. Mazella, E. Holland, and S. Rao (1996), Algorithms that use the ionosphere to control GPS errors, paper presented at Position Location and Navigation Symposium, Inst. of Electr. and Electron. Eng., Atlanta, Ga., 22-26 April. Brunini, C., A. Meza, and W. Bosch (2005), Temporal and spatial variability of the bias between TOPEX- and GPSderived total electron content, J Geod., 79, 175-188.

Chen, W., C. Hu, S. Gao, Y. Chen, and X. Ding (2004), Absolute ionospheric delay estimation based on GPS PPP and GPS active network, paper presented at International Symposium on GNSS/GPS, Sydney, Australia, 6-8 Dec.

Coco, D. S., C. Coker, S. R. Dahlke, and J. R. Clynch (1991), Variability of GPS satellite differential group delay biases, IEEE Trans. Aerosp. Electron. Syst., 27, 931-938.

Foster, J. C., and W. Rideout (2005), Midlatitude TEC enhancements during the October 2003 superstorm, Geophys. Res. Lett., 32, L12S04, doi:10.1029/2004GL021719.

Goodwin, G. L., and A. M. Breed (2001), Total electron content in Australia corrected for receiver/satellite bias and compared with IRI and PIM predictions, Adv. Space Res., 27(1), 49-60.

Grejner-Brzezinska, D. A., P. Wielgosz, I. Kashani, D. A. Smith, and P. S. J. Spencer (2004), An analysis of the effects on different network-based ionosphere estimation models on rover positioning accuracy, J. Global Positioning Syst., 3, $115-131$.

Hernandez-Pajares, M., J. M. Juan, J. Sanz, and R. Orus (2004), Wide area real time kinematics with Galileo and GPS signals, paper presented at ION GNSS 17th International Technical Meeting of the Satellite Division, Long Beach, Calif., 21-24 Sept.

Jakowski, N., E. Sardon, E. Engler, A. Jungstand, and D. Klahn (1996), Relationships between GPS-signal propagation errors and EISCAT observations, Ann. Geophys., 14, 14291436 .

Kee, C., and D. Yun (2002), Extending coverage of DGPS by considering atmospheric models and corrections, J. Navig., 55, 305-322, doi:10.1017/S0373463302001741.

Komjathy, A. (1997), Global ionospheric total electron content mapping using the Global Positioning System, Ph.D. thesis, Dep. of Geod. and Geomat. Eng., Univ. of N. B., Fredericton, Canada.

Komjathy, A., and R. Langley (1996), An assesment of predicted and measured ionospheric total electron content using a regional GPS network, paper presented at Natl. Tech. Meet., Inst. of Navig. Santa Monica, Calif., 22-24 Jan.

Lanyi, G. E., and T. Roth (1988), A comparison of mapped and measured total ionospheric electron content usin Global Positioning System and beacon satellite observations, Radio Sci., 23, 483-492.

Leick, A. (2004), GPS Satellite Surveying, 3rd ed. John Wiley, Hoboken, N. J.

Liao, X. (2000), Carrier phase based ionosphere recovery over a regional area GPS network, M.Sc. thesis, Univ. of Calgary, Calgary, Alberta, Canada.

Lin, C. H., A. D. Richmond, J. Y. Liu, H. C. Yeh, L. J. Paxton, G. Lu, H. F. Tsai, and S.-Y. Su (2005), Large-scale variations 
of the low-latitude ionosphere during the October-November 2003 superstorm: Observational results, J. Geophys. Res., 110, A09S28, doi:10.1029/2004JA010900.

Lin, L. S. (2001), Remote sensing of ionosphere using GPS measurements, paper presented at the 22nd Asian Conference on Remote Sensing, 5-9 Nov., Singapore.

Ma, G., and T. Maruyama (2003), Derivation of TEC and estimation of instrumental biases from GEONET in Japan, Ann. Geophys., 21, 2083-2093.

Makela, J. J., M. C. Kelley, J. J. Sojka, X. Pi, and A. J. Manucci (2001), GPS normalization and preliminary modeling results of total electron content during midlatidute space weather event, Radio Sci., 36, 356-361.

Mitchell, C. N., L. Alfonsi, G. De Franceschi, M. Lester, V. Romano, and A. W. Wernik (2005), GPS TEC and scintillation measurements from the polar ionosphere during the October 2003 storm, Geophys. Res. Lett., 32, L12S03, doi:10.1029/2004GL021644.

Nayir, H. (2007a), Instrumental bias estimation using single station GPS/TEC, paper presented at IRI/COST 296 Workshop. Prague, Czech Republic, 10-14 July.

Nayir, H. (2007b), Ionospheric total electron content estimation using GPS signals (in Turkish), M.Sc. thesis, Hacettepe Univ., Ankara, Turkey.

Nayir, H., F. Arikan, O. Arikan, and C. B. Erol (2007), Total electron content estimation with Reg-Est, J. Geophys. Res., 112, A11313, doi:10.1029/2007JA012459.

Otsuka, Y., T. Ogawa, A. Saito, T. Tsugawa, S. Fukao, and S. Miyazaki (2002), A new technique for mapping of total electron content using GPS network in Japan, Earth Planets Space, 54, 63-70.
Sardon, E., A. Rius, and N. Zarraoa (1994), Estimation of the receiver differential biases and the ionospheric total electron content from Global Positioning System observations, Radio Sci., 29(3), 577-586.

Schaer, S. (1999), Mapping and predicting the Earth's ionosphere using the Global Positioning System, Ph.D. thesis, Univ. of Bern, Bern, Switzerland.

Warnant, R. (1997), Reliability of the TEC computed using GPS measurements - The problem of hardware biases, Acta Geod. Geophys. Hung., 32(3-4), 451-459.

Yizengaw, E., M. B. Moldwin, P. L. Dyson, and T. J. Immel (2005), Southern Hemisphere ionosphere and plasmasphere response to the interplanetary shock event of 29-31 October 2003, J. Geophys. Res., 110, A09S30, doi:10.1029/ 2004JA010920.

Zhang, Y., F. Wu, N. Kubo, A. Yasuda (2003), TEC measurement by single dual-frequency GPS receiver, paper presented at International Symposium on GPS/GNSS, Tokyo, Japan, 15-18 Nov.

F. Arikan and U. Sezen, Department of Electrical and Electronics Engineering, Hacettepe University, Beytepe, 06532 Ankara, Turkey. (arikan@hacettepe.edu.tr; u.sezen@ee. hacettepe.edu.tr)

O. Arikan, Department of Electrical and Electronics Engineering, Bilkent University, Bilkent, 06533 Ankara, Turkey. (oarikan@ee.bilkent.edu.tr)

H. Nayir, Department of Microwave and System Technologies, Aselsan Inc., Yenimahalle, 06370 Ankara, Turkey. (hnayir@mst.aselsan.com.tr) 